\title{
Natural language processing in mining unstructured data from software repositories: a review
}

\author{
SOM GUPTA $^{1, * \mathbb{D}}$ and S K GUPTA ${ }^{2}$ \\ ${ }^{1}$ Department of Computer Science Engineering, AKTU, Lucknow, India \\ ${ }^{2}$ Department of Computer Science Engineering, BIET, Jhansi, India \\ e-mail: somi.11ce@gmail.com
}

MS received 16 June 2017; revised 15 March 2019; accepted 7 September 2019

\begin{abstract}
With the increasing popularity of open-source platforms, software data is easily available from various open-source tools like GitHub, CVS, SVN, etc. More than 80 percent of the data present in them is unstructured. Mining data from these repositories helps project managers, developers and businesses, in getting interesting insights. Most of the software artefacts present in these repositories are in the natural language form, which makes natural language processing (NLP) an important part of mining to get the useful results. The paper reviews the application of NLP techniques in the field of Mining Software Repositories (MSR). The paper mainly focuses on sentiment analysis, summarization, traceability, norms mining and mobile analytics. The paper presents the major NLP works performed in this area by surveying the research papers from 2000 to 2018. The paper firstly describes the major artefacts present in the software repositories where the NLP techniques have been applied. Next, the paper presents some popular open-source NLP tools that have been used to perform NLP tasks. Later the paper discusses, in brief, the research state of NLP in MSR field. The paper also lists down the various challenges along with the pointers for future work in this field of research and finally the conclusion.
\end{abstract}

Keywords. Natural language processing; mining unstructured data; software artefacts; software repositories; mobile analytics.

\section{Introduction}

During software evolution and maintenance, which accounts for almost 90 percent of software resources [1], the developers and the businesses need to look at the various software artefacts like requirement document, bug reports, commit messages, etc. present in the software repositories. They are mostly in the natural language form and contain unstructured data. For example, the requirement document generated at the analysis phase is textual and contains lot of information about the project in it; bug reports produced during testing time contain insights about the quality of product and indicates the effectiveness of testing; commit messages in the version systems describe the code changes and why they are made. Mining these repositories not only helps get insights about the processes but also helps in decision-making in terms of both the product and process for further enhancements and improvements.

Unstructured data is data that has no specific format and no data model. Text data, image data and video data are some of the examples of unstructured data. This type of

*For correspondence

Published online: 30 November 2019 data is estimated to represent 80 percent of the valuable information for most of the organizations.

With the emergence and popularity of open-source systems, the number of online repositories has increased in previous years, which has attracted the attention of many researchers as they serve as a great resource of software engineering data. There are a number of publicly available open-source repositories like GitHub [2], SourceForge [3], Google code [4], Bugzilla [5], etc. Also, the question-answering sites like StackExchange [6], StackOverflow [7], etc. contain valuable information about the software engineering data. By the end of 2017, GitHub had 24 million users, 1.5 million active organizations, more than 67 million repositories [8]; around 1 billion commits, 25.3 million active repositories and 12.5 million issues by the end of September 2016 [8]. This software engineering data is growing exponentially year by year. This data presents immense opportunities to researchers to find out the interesting insights or patterns from them.

Mining Software Repositories (MSR) is the process of analysing and extracting the knowledge and patterns from the software artefacts, which are available in the software repositories in the archived form to support the decisionmaking and improve the software processes. Many times, more than 1 software repositories are integrated to get 
useful and interesting results. MSR transforms the static repositories into active repositories. Mining repositories helps software teams understand the projects better, and thus leads to high-quality and cost-effective project development [9].

Manually going through each of the artefacts to gather the required data is not an easy task. It is a time-consuming process. Thus, automation is performed via automatic summarization, automatic sentiment analysis, traceability analysis, mining, preprocessing, etc. However, noisy, incomplete and ambiguous data pose challenges in performing these tasks automatically.

In order to perform the afore-mentioned tasks, data mining, text analytics, information retrieval and natural language processing (NLP) are applied. Due to the fact that most of the artefacts contain natural text that is heterogeneous and noisy, NLP plays a very important role in analysing these artefacts. Further, software data is very complex and analysing it requires knowledge about the semantics and context [10]. NLP is a computerized approach to analyse, understand and find the meaning of text free of natural language. It analyses the various aspects of language and helps about the questions related to why, who, where, whom and how. It includes many techniques like part of speech (POS) tagging, semantic dependency graph representation of text, named entity recognition, word sense disambiguation, parsing, etc. NLP helps perform spelling correction, stemming, abbreviation expansion, concept location, text summarization, sentiment analysis, autocompletion in IDE, topic segmentation, question-answering [11], etc.

The paper reviews the application of NLP in software repositories. It focuses on its application in sentiment analysis, summarization, mobile analytics, norms mining and traceability. In this paper, we have investigated the studies in the field of MSR where the NLP techniques have been used to get the answer to these questions:

RQ1: What are the major artefacts where the NLP techniques have been used and where there is a scope of further research?

RQ2: What are the various types of applications that can be performed by NLP techniques?

RQ3: What are the various tools/techniques being used to perform the various applications in software repositories?

RQ4: What are the challenges associated with using NLP techniques in these repositories?

RQ5: Is NLP alone sufficient to perform these applications or are there some limitations?

RQ6: What are the areas where there is a need of attention to researchers?

The paper is organized as follows. Section 2 states the natural language artefacts present in the repositories. Section 3 gives an overview of NLP techniques and application of open-source NLP tools. Section 4 discusses the applications and related work of NLP in software repositories. Section 5 discusses the strengths and limitations of NLP in MSR. Section 6 discusses the methodology used for choosing the papers. Section 7 gives a brief analysis on the research trends. Section 8 discusses the future scope of NLP in software repositories and finally the conclusion.

\section{Natural language artefacts in software repositories}

There are a number of software artefacts that are created during the software development process in order to share the knowledge. Discussion among team members is either through chats or e-mails, and is mostly textual. These artefacts and discussions are stored in the software repositories for further reference and use. Most of the artefacts contain natural language text in them. These documents serve as a great resource of knowledge and contain a lot of information related to project understanding, project evolution and project planning. From the recent papers published, we have observed that most of the research in NLP in this field is focused on software process documents, archived communications, discussions in question-answering sites, source code and mobile app store reviews.

There are a number of software process documents that are created during various phases of software life cycle. During requirement phase, requirement document is created, which contains functional and non-functional requirements of the software in consultation with the customers. They are produced at the first stage of software development, and at this stage the errors are the cheapest and easiest to correct. NLP can be used to extract ontologies from requirements specifications and verify the consistency or completeness of a requirement specification. NLP techniques help classify and prioritize the requirements [12].

During design phase, design documents are created, which include architecture design document, database design document, technical documents (which describe algorithms, interfaces, etc.), etc. These documents contain both the graphical images (UML diagrams) for the designs and natural text. Mining the natural text from these documents helps get important information about a project like the design patterns, the software use, knowledge discovery about the project [13], etc.

During testing phase, bug reports are created, which contain a number of fields that include natural language text like title, description and comments. Bug reports contain discussion among multiple people about a particular issue in the comments section [14]. In open-source projects, bug repositories play a very important role as they allow people to report the defects they observe. The reports include not just defects but also sometimes the desired enhancements 
that can be implemented in a system. While making changes to the software and releasing a software, commit logs and release notes are created [15]. Version control systems are used by developers mainly to describe the changes to the systems in commit messages. Commit messages are mostly textual and describe the code changes. Many times, developers mention why they made those particular changes. With NLP, "why" side of a commit message can be analysed. Analysing the "why" side of the software helps get the valuable information for software maintenance purpose, and can help in formulating the golden rules for the coming projects. Release note [16] summarizes the changes that occurred in a software system from its previous release. It includes information such as the enhancements in the system, license changes, code changes, document changes, re-factoring operations, etc. They are mainly written in the natural language text.

In large software development teams, members are geographically dispersed and their main source of communication is e-mails and chats. Along with the reference to formal documents, to clear their doubts and for better understanding, developers discuss the documents, their expertise, code issues, solutions to bugs, team dynamics, etc. through these informal modes of communication. Mining this informal mode of information helps get very useful information like collaboration style of team, which can help managers take decisions related to team structure and task allocation.

Question-answering sites like StackOverflow [7] and Stack Exchange [6] allow the users to post questions and receive the solutions. A user's post may contain both the textual description and code fragments. Mining textual information helps perform some interesting tasks like categorizing the questions, recommending tags for a question, finding the sentiments, etc.

Source code is also one of the very popular NLP components for mining information, which contains comments, string literals and variable names, which are in the textual format. Modules, classes and methods contain attributes like author name, brief description and a date when the artefact was created. Using this information, many interesting things have been mined like finding semantically similar words, finding the responsibilities of methods [17], etc.

With the tremendous growth of mobile industry, and increasing use of smart phones, the number of mobile apps coming every day is increasing very fast. As of March 2018, the number of mobile apps in Google Play Store was around 3.8 million and the number of mobile apps in Apple Play Store was around 2 million [18]. These figures of the number of mobile apps in these markets create an immense opportunity for researchers to mine the information from them to get useful insights, which can help both developers and users. These app stores contain Rating and Review mechanism for each app. Review data is in natural language and thus, with NLP, mining becomes easier.

\section{NLP tools and techniques: a brief introduction}

The knowledge of language falls under the levels of [20]:

- Phonology and phonetics, to study how the words are pronounced.

- Morphology, to study the internal structure of the word and to find out how the word is made up from smaller meaningful words like finding stem words, or prefixes or suffixes for a word. It is widely used in text for speech synthesis, information retrieval, machine translation and grammar correction.

- Syntax, to find out how the words group together and what relations they share.

- Semantics, to find the relation between the symbols and reasoning with meaningful representation of natural language, and falls into: lexical semantics, which is to understand the meaning of components of words, and distributed semantics, which is to study how the word components group together to form larger meanings. It finds its application in query expansion where the relevant words for a query can be identified, analogy testing, finding relationships between the words, word sense disambiguation, etc.

- Pragmatics, to study how the language can accomplish a goal.

- Discourse, to interpret the next sentence by understanding the preceding sentence.

Mostly state machines and formal rules are used for the phonology and phonetics, morphology and syntax. State machines include finite-state automata (FSA), weighted automata, finite-state transducers, Markov models and hidden Markov models. Formal rules include contextfree grammars (CFGs), and the variants like probabilistic CFGs and feature-augmented grammars. State machines and formal rules use graph algorithms like DFS (depth first search), BFS (breadth first search), A* and dynamic programming approach. FSA act as language recognizers whereas transducers convert string of one language to another. Probabilistic hidden Markov models are used for POS tagging. CFGs are used for syntax parsing for a sentence. Dependency graphs are used for representing the relations between the words in a sentence.

Mostly predicate calculus, semantic networks, conceptual dependency and feature structures are used for semantics, discourse and pragmatics. Vectors are used for representing the features.

There are many NLP tools that help perform these NLP tasks. Some famous open-source NLP tools along with the functionality are given in table 1 .

We have also identified the various techniques used for performing some famous NLP tasks. Table 2 shows the various NLP applications and major techniques used for performing them. 
Table 1. Open-source NLP tools and their functionalities.

\begin{tabular}{|c|c|}
\hline Tool name & Functionalities available \\
\hline Apache & It is a Java-based machine learning toolkit used for processing natural-language-free text. It helps \\
\hline OpenNLP & $\begin{array}{l}\text { in sentence detection, tokenization, de-tokenization, named entity recognition, documents categorization, POS } \\
\text { tagging, chunking and co-reference resolution. It also supports maximum entropy machine learning [23]. }\end{array}$ \\
\hline Stanford & It is a set of natural language analysis tools. It helps perform a number of operations like POS \\
\hline CoreNLP & $\begin{array}{l}\text { tagging for a sentence, named entity recognition, co-reference resolution, bootstrapped pattern learning, etc. It gives } \\
\text { support for a large number of languages and their interface is available for many modern programming languages } \\
\text { [24]. }\end{array}$ \\
\hline Python NLTK & $\begin{array}{l}\text { NLTK is a python package that implements many standard NLP data structures and algorithms. } \\
\text { It supports tokenization, classification, stemming, tagging, chunking text, sentiment analysis, semantic reasoning, } \\
\text { etc. [25]. }\end{array}$ \\
\hline GATE & $\begin{array}{l}\text { It is an open-source Java-based text analysis tool. It helps perform a number of NLP-based tasks } \\
\text { like tokenization, sentence-splitting, POS tagging, entity names identification, semantic tagging, anaphora } \\
\text { resolution, etc. [26]. }\end{array}$ \\
\hline spaCy & $\begin{array}{l}\text { It is an open-source industrial strength NLP tool. It helps perform a number of NLP tasks like } \\
\text { tokenization, named-entity recognition, POS tagging, lemmatization, dependency parsing, similarity calculation, } \\
\text { rule-based matching, etc. It helps perform deep learning too [27]. }\end{array}$ \\
\hline EmoTxt & $\begin{array}{l}\text { Toolkit for emotion recognition. It finds positive and negative emotions, and also the emotions in } \\
\text { text [28]. }\end{array}$ \\
\hline MALLET & $\begin{array}{l}\text { Java-based library for statistical NLP. It includes features like text classification, topic } \\
\text { modelling, sequence tagging [29]. }\end{array}$ \\
\hline KERAS & $\begin{array}{l}\text { Deep learning platform that works using TensorFlow [30], CNTK [31] and Theano [32] as the } \\
\text { back-end. It helps build the deep neural network, convolutional networks, recurrent networks and helps in transfer } \\
\text { learning for NLP [33]. }\end{array}$ \\
\hline
\end{tabular}

Table 2. Applications and techniques used.

\begin{tabular}{|c|c|}
\hline Application & Techniques used \\
\hline Text pre-processing & $\begin{array}{l}\text { Tokenization, stemming, stop word removal, identifier splitting, pruning, abbreviation } \\
\text { expansion, synonyms identification }[15,34]\end{array}$ \\
\hline Spelling Correction & Minimum edit distance, $n$-gram models \\
\hline POS tagging & $\begin{array}{c}\text { Hidden Markov models (Viterbi decoding, Baum-Welch algorithm), maximum entropy } \\
\text { models, conditional random fields }\end{array}$ \\
\hline Dependency parsing & Feature models, rich semantic graphs [35], spanning trees, AMR graphs [36] \\
\hline Word embedding & Word2Vec [84], CBOW, Skip Gram models, Hellinger PCA [21] \\
\hline Similarity calculation & $\begin{array}{l}\text { Cosine measures, dice, jaccard [34], path-based similarity, Leacock-Chodorow (L-C) } \\
\text { similarity, Jiang-Conrath (J-C) distance-based similarity, Extended Lesk algorithm, concept } \\
\text { probability models }\end{array}$ \\
\hline Word sense disambiguation & $\begin{array}{l}\text { Lesk algorithm, Walker algorithm, random walk algorithms, naive Bayes, decision list } \\
\text { algorithms, bootstrapping, Yarowsky's method, hyperlex [37] }\end{array}$ \\
\hline Text summarization & $\begin{array}{l}\text { Term frequency-inverse document frequency, PageRank, AMR graphs [36], rich } \\
\text { semantic graphs [35], entities extraction, content clustering, Lesk calculation }\end{array}$ \\
\hline $\begin{array}{l}\text { Sentiment analysis and } \\
\text { opinion mining }\end{array}$ & $\begin{array}{l}\text { Naive Bayes, SVM, maximum entropy, POS tagging, dependency analysis, negation } \\
\text { handling, } n \text {-gram model [38] }\end{array}$ \\
\hline Topic models & Latent Dirichlet Allocation (LDA) and its variants \\
\hline Source code analysis & Syntax trees [17], $n$-gram models, smoothing algorithms \\
\hline Text classification & Naive Bayes, logistic regression, SVM, Word2Vec [38], convolutional neural network \\
\hline
\end{tabular}

\section{Applications and research state of NLP in software repositories}

\subsection{Bug repositories processing}

Bug repositories contain the issues along with their solutions, which help understand the context of bug and thus, help during software development, maintenance and evolution [39]. Assignment of a bug to a proper person according to the severity and other aspects is one of the main challenges for a project manager. Many times the bug reports are either duplicate or their content is irrelevant. NLP helps ensure the quality of bug reports and improve prioritization. NLP helps improve the quality of mining 
results from bug repositories and thus helps in classification of bug reports. Schugerl et al [39] proposed an approach to automatically evaluate the quality of bug reports by analysing the natural language bug description and comments present in them. They evaluated the free-form description of bug reports on the basis of attributes like reproducibility, observability, certainty and focus. They used NLP techniques to find out the semantic information from the documents.

Most of the time, an open-source project maintains an open bug repository, which allows the people all over the world to report the anomaly or any bug they observe. When persons observe some bugs, they submit it to the bug repository and then the bug goes for approval from a person called triager, who checks if the bug posted is duplicate or not. However, given the scenario that it is open for everyone, the number of reports usually grows up tremendously and thus makes it very challenging for the triager to examine all the bug reports to detect duplication. Duplicate bug reports have been identified automatically by applying NLP, which helps minimize the load of triagers.

Runeson et al [34] used the NLP techniques to detect the duplicate bug reports. They used tokenization, stop word removal and stemming for preprocessing of defect reports and then used vector space model along with the cosine measure for measuring the similarity between the defect reports. In 2010, Surekha and Jalote [40] used the character $n$-grams to detect the duplicate bug reports. Minh [41] used both character $n$-gram model and word-level $n$-gram model to detect the duplicate bug reports. Banerjee et al [42] proposed an approach to analyse the impact of problem of alternate spelling, typographical errors and synonyms in the open-source bug repositories to find out the duplicate bug reports. They used Levenshtein distance analysis to find the similarity between the text sequences and then used neighbour word likelihood measures. In another paper, Banerjee et al [43] used the sequence matching and string matching to find the duplicate bug reports.

\subsection{Improve software evolution and maintenance}

Globally distributed teams, complexity of projects in terms of size and resources, and continuous change of requirements lead to some challenges during evolution and maintenance phase. It has been found that sometimes the cost of software maintenance goes to 70 percent of the total cost of software project [44]. Source code is the major component that is produced during software development. It contains variable names and comments in it, which are mostly written in natural language text. The study of quality of variable names and comments in the code using NLP techniques will help software project managers improve the quality of natural text in the source code. Good qualities of code comments and variable names help the other members of the team understand the project easily and thus reduce efforts during software evolution or maintenance. Source code summarization creates automatic documents and helps in program comprehension, which is of help during the evolution and maintenance phase [45].

\subsection{Sentiment analysis}

Sentiment analysis is to find out the positive, negative or neutral attitude of a user. It has been applied to various types of businesses like movies, education, hotels, mobile industry, social media, blogs, stock markets, news articles, etc. to analyse the sentiments through user's reviews. Due to the collaborative nature of software process, software artefacts contain a lot of information about the human efforts and interaction, so they are likely to contain substantial amount of emotions. The main task of sentiment analysis is to find out the opinions, identify the sentiments and classify them according to their polarity [46].

Sentiment analysis in the field of software engineering can help devise measures to boost up the morale of team, job satisfaction and better job assignment. The sentiment analysis in this field is mostly focused on question-answering or discussion sites, commit messages, e-mail threads and mobile app user's reviews. In commit messages, sentiment analysis can help managers understand the morale of development team. In large companies and mostly in open-source projects, people are dispersed geographically and it is said that the success of a project depends upon the morale of team. With sentiment analysis of e-mail threads, the morale of team can be found, which can prove to be very useful for a manager as productivity of team increases when members of team are satisfied and happy [44, 47]. Sentiment analysis is an NLP application, which involves expansion of contractions like I'm to I am, identifying the emoticons, negation processing, stemming, stopping word removal, etc. for analysis.

Discussion sites generate question-answers that are in the natural text. With sentiment analysis, best answer can be predicted. Sentiment analysis has been applied in many applications in software engineering domain like mobile analytics, to detect the polarity of mobile app user's reviews. Sentiment analysis in mobile analytics helps know developers more about their app from customer's acceptance perspective. Goul et al [48], in their paper, investigated how the sentiment analysis can help in requirement engineering in mobile apps. In another study by Carreno and Winbladh [49], they analysed the polarity in the mobile app reviews along with the topic modelling to extract the requirements for next version of app.

Tourani et al [47] analysed the sentiment values in mailing lists of apache project Tomcat [50] and ant [51] using SentiStrength tool. They used e-mails of both users (customer support communications) and developers (communication among software team). They categorized the sentiments into positive (satisfactory-opinion, friendly- 
interaction, explicit-signals or emoticons, socializing or mails describing events or meetings, curiosity or mails asking questions with positive sentiments), negative (aggression, that is, mails with problems and complaints, uncomfortable situation, sadness) and neutral.

Bazelli et al [52] determined the author's personality traits using linguistic and word count tool, which has a dictionary in it. They used the dictionary of 2700 words. Authors were classified on the basis of reputation, post content and post votes. They classified personality traits for authors into the following five: Neuroticism, Extroverts, Openness, Agreeableness and Consciousness.

Ortu et al [53] and Murgia et al [54], in their papers, analysed how the sentiments in bug reports impact the bug fixing time. They found that positive sentiments lead to short fixing time whereas negative sentiments lead to long fixing time. Ortu et al [55], in another paper, analysed the issues or bug reports in issue tracking system and labelled the emotions to create a resource for further research in software engineering community.

Islam and Zibran [56] created a tool called SentiStrength-SE, which is an extension of SentiStrength tool to improve accuracy in the context of software engineering domain.

Guzman et al [57] analysed the developers' emotions in the commit messages and analysed how the sentiments are related with programming language, day of work and team distribution. Sinha et al [58] extended the work by Guzman et al [57] and analysed the sentiments from the commit logs. They answered questions such as the following: what are the general developer's sentiments in GitHub projects, how are the day of the commit message and the sentiment related and how does the sentiment differ with number of changes performed? Calefato et al [59] have developed an approach called Senti4SD, which uses lexicon-based analysis, word embeddings for semantic analysis and keywordbased features to find the polarity of emotions.

\subsection{Summarization}

This is to reduce the size of a development artefact without losing the main information content about it. When a user types the query, the system retrieves the documents and the document size can be anything. For a person to read all the documents is a difficult task. It has been observed that during maintenance phase, 60 percent of the time is spent reading or studying the software [60]. Hence, summarization can prove to be a good solution to the problem. Summarization is of two types: extractive and abstractive. Extractive summarization [61, 62] is to extract the important sentences from the document and arrange them in the way they appear in the original document, whereas abstractive summarization [62] is to select the important phrases or sentences and then arrange them in meaningful order by applying linguistic rules. NLP plays an important role in the abstractive summarization as it requires the knowledge about the syntax, semantics and context and this is possible from NLP techniques only [61, 63]. The common artefacts where the summarization has been applied are the following.

4.4a Bug reports: Summarizing the bug reports helps developers to know the essence of bug report to efficiently determine whether it contains the required information or not. Concise automatic summaries so produced will also help developers navigate the bug reports quickly and thus find the required information quickly [14]. It will reduce the developer's time and effort while reviewing the bug reports [64].

Nithya and Arunkumar [64] proposed a system that summarizes the bug reports using feature extraction. They pass the bug reports to the noise reduction module, which categorizes the sentences on the basis of calculated feature values, and then use this for creating the summary.

Rastkar et al [14] proposed an approach to automatically generate bug reports summary. They used the existing conversation-based techniques, which are generally used for e-mail threads and other conversations, using binary classifiers based on features.

Lotufo et al [65] used textual similarity metrics like Levenstein edit distance and euclidean distance to measure the similarity of contents. They used tf-idf and porter stemming before converting the sentences to the vector form for further processing. Polarity detection through sentiment analysis was used to evaluate the relations between sentences in a bug report.

$4.4 \mathrm{~b}$ Source code: During the maintenance phase of software, developers often need to understand the software project. However, given the scenario that developers keep on changing in large software development teams and the teams are usually large, it becomes difficult to understand the whole source code. In these situations, source code summarization reduces the time to navigate the whole source code and helps them understand the project easily. Manual summarization is time-consuming and error-prone. It has been observed that most of the time, source code summaries are incomplete. Automatic source code summarization is to create a brief summary of the source code that describes the methods, functions, calls, etc. It relieves the developers from the tedious work of documentation [66].

The work in the field of source code summarization started from the work of Buse and Weimer [60], where they used symbolic information to create the template-based abstractive summaries to find the causes of an exception that a particular method can raise. Sridhara et al [1] tried to overcome the domain-dependency shortcomings of the work by Buse et al [60], by generating descriptive source code comments utilizing the signature and body of method. Later, Haiduc et al [66] used a combination of position of terms in the software and text retrieval techniques to find 
out the meaning of methods and classes in the source code to create the automatic source code summaries. Rastkar et al [17] introduced an approach to automatically generate the natural language summaries for cross-cutting source code concerns (that is, when the code to be changed is not from just one module but multiple modules in the project) using the information from identifier names in the concerning code, description of natural language present in the source code and salient code elements. Summaries so produced by their approach include listing of methods where changes need to be done for a particular change concerned, a short summary of concern, dependent code elements and textual description of the path patterns.

McBurney and McMillan [63], in one of their papers, described the usage of Software Word Usage Model as call graph generator, PageRank and natural language generation to create context-based source code summaries. They analysed the internal working of methods, their usage and purpose of their existence to create the summaries. Guerrouj et al [67] generated natural language summaries for source codes for stackOverflow discussions by extracting the library identifiers from free-form natural language text using island parsers. Chitti Babu et al [68] used the NLP techniques to extract the entities to perform source code summarization.

Source code summarization also helps in creating automatic documents. Source code has been used in automatically generating the release notes and commit messages.

- Automatic generation of release notes

Release notes are generated by applying summarization techniques on source codes and then linking them with issue tracking systems. Release notes contain information about all changes that are implemented in the new version of the software with respect to the previous version. It includes all kind of changes, including the code changes, new functionalities added and the functionalities dropped, open issues and closed issues, document changes, re-factoring operations, license changes, library changes [16], etc.

Moreno et al [16] proposed an approach called ARENA, i.e., Automatic Release Notes Generator, to automatically generate the release notes by summarizing code changes and then linking these changes to the commit notes and issue trackers. They used Vector Space Model and cosine similarity measures for computing the textual similarities between the commit notes and issues.

- Automatic generation of commit messages

Commit messages tell about the changes that have been made and the reasons for which they are made. However, it is observed that most of the times the commit messages are either incomplete or empty. Commit messages play an important role during software evolution. They help understand the software from the changes point of view. Developers are usually very busy doing coding and other tasks, so they do not take the commit messages very seriously, which results in the poor quality of commit messages. Automatic creation of commit messages using summarization techniques helps maintain good quality of commit messages [69]. Cortes-Coy et al [70] proposed an approach called ChangeScribe, which generates the commit messages automatically by summarizing source code changes between the two versions of software, namely old and new versions of the software, by extracting the change set between the two versions and finding the responsibilities of methods in the class. Shen et al [45] analysed the type of methods and type of maintenance tasks in the source code along with the stereotypes, to generate the commit messages.

- Automatic unit test case generation

Maintaining unit test cases is a very time-consuming task, but it is very essential during software evolution and maintenance. They help during regression testing, maintaining legacy code and improving the quality of code. Automatic unit test case generation reduces the burden on developers [71]. Li et al [71] proposed an approach called UnitTestScribe to automatically generate the natural language documents of unit test cases by identifying the focal methods and then created the template-based abstractive summaries [62].

4.4c Heterogeneous data in question-answering sites: Ponzanelli et al [72] proposed a technique to summarize stackOverflow discussions. Their approach dealt with heterogeneous data of stackOverflow that has natural language description about the discussions, code segments, XML data, etc. They extended the LexRank approach and devised a custom similarity function for this type of data. Even Islam and Zibran [56], in their paper, stated the difficulty of separating the source code from textual data.

\subsection{Traceability}

In incremental software development models, at the end of each phase, some module of the project is released. At the beginning of each cycle, either the new functionality is implemented or the enhancements are done by doing some modifications to the existing functionality. As the software evolves, the number of software artefacts increases and managing them becomes a challenge. When a change has to be implemented, the change sometimes causes the ripple effect. To resolve it, developers have to go through various documents to understand the impact of changes on the other documents. By NLP, we can extract information from various artefacts and thus can help automatically maintain the traceability links using some tools. This will reduce the developer's time of managing and tracing the artefacts. 
This will improve the software process and thus reduce the development time, assist in change management and scope management, and hence increase the quality of a project [73]. Aponte and Marcus [74] used the NLP techniques to create the human readable summaries.

Alobaidi and Mahmood [73] found the relationship between requirement documents and others using semantic similarity and semantic relatedness. Arunthavanathan et al [75] used the NLP techniques to convert the natural language requirement document into XML format by extracting classes, methods, attributes and relationships and then used the XML format so generated in their tool "Software Artefacts Traceability Analyser (SAT)," which resolved the problem of artefacts traceability, that is, links between the artefacts like Requirements, UML Class Diagrams and Java code of the project, when a change occurs. Le et al [15] proposed the approach called RCLinker, where they found the link between the issue reports and the commit messages by considering the context along with comparing the text between these two artefacts.

\subsection{Preprocessing of unstructured data}

Be it the summarization technique, sentiment analysis, norm mining or any kind of mining in software repositories, preprocessing is the must step. Without preprocessing, it is difficult to get meaningful results. Mainly in discussion sites, bug repositories and mobile app reviews, which are online and freely allow the users to post data, use of slangs, abbreviations and spelling mistakes are very frequent. These problems, if not handled properly, mislead the model and also reduces the performance of model. POS tagging, namedentity resolution, pronoun resolution, obtaining predicateargument structure, dependency parsing, etc. are a few of the very common things that are done before performing the NLP task. There are many NLP tools available to perform these tasks, like WordNet [76], PropBank [77], NLTK [25], Stanford NLP [24], VerbNet [78], JAMR Parser [79], etc.

In order to understand the semantics and features of any text, which is the basis of performing further mining activities, many NLP techniques are used, like POS tagging, construction of dependency trees, construction of word graphs, abstract meaning representation (AMR) graphs, rich semantic graphs (RSG), etc. [80]. In the paper by Gupta and Gupta [80], they mention how these approaches have been successfully used for the summarization approach and similarly they can be used for other activities too.

Santos and Ladeira [81], in their paper, analysed the impact of text preprocessing before analysing the data, and found that preprocessing improved the precision of POS tagger. Banerjee et al [43] used the NLP steps like stemming, tokenization, stop word removal and pruning to reduce the language space for finding the duplicate bug reports.

McIlroy et al [82] created an automatic approach to label the issues raised by users in their reviews in the categories like feature request, network problem, update issues, response time issue, user interface issue, etc. They used the machine learning approach (ML approach) to perform this task. However, without proper preprocessing (abbreviation handling, handling of incorrect grammars, handling of slangs and segmentation into sentences) using natural language techniques, the ML approach would not have worked efficiently.

Hu et al [83], in their paper, to extract the discussion topics using LDA, preprocessed the data to get the meaningful results. They changed all the words to lower case, removed punctuation marks and stop words, and performed porter stemming to stem user reviews. Vu et al [84] preprocessed the user's reviews from Google Play to extract the keywords for the purpose of mining opinions.

\subsection{Mobile analytics}

With growing popularity of mobiles, number of apps coming up is increasing day to day. Normally a user reviews the app using rating and textual description. This textual description gives more insights about the mobile app than the ratings [85]. Users post their views about the app in the reviews. They post the problems they face while using the app, any bug that they find, their praise for app, privacy issues [82] and sometimes suggestions for what more they need in the app. NLP techniques play a very important role in summarizing and finding sentiments like finding user's level of satisfaction or mining information from them. Without NLP, the results from any technique will not be fair because of the noisiness in the text like spelling mistakes, incomplete sentences, use of jargons, etc. NLP techniques have been used in many applications in mobile analytics like energy assessment in mobile apps by mining permissions in the mobile apps, finding the complementary or derogatory parts of mobile app. App market also allows the developers to add versionspecific text when the new version has to be added; this change information can prove to be useful for getting information related to evolution of the app.

Iacob and Harrison [86] designed a prototype called MARA, which is Mobile App Review Analyser. It can automatically retrieve the online reviews about the app, mine them by identifying the sentences and present them in a user-friendly manner. According to Gao et al [87], MARA's approach to extract features on the basis of keywords alone does not work on practical analysis. Thus, to address this issue, they created a framework called ARTracker, which extracts topics from the user review's data through various topic models like Latent Dirichlet Allocation (LDA), Latent Semantic Indexing (LSI), Random Projection (RP), Gibsampling LDA and Non-Negative Matrix Factorization (NMF), and then ranked them according to the importance. Liu et al [88], in their paper, proposed an approach based on sentiment analysis to summarize the user reviews in app store. Hu et al [83] also 
used Twitter LDA to extract the discussion topics of crossplatformed hybrid apps. Vu et al [84] created a web-based tool called MARK, which is Mining and Analysing Reviews by Keywords, which supports the analysis of app's reviews based on the keywords. Cheng et al [89] used Markov transition models for creating an app recommendation approach by considering the short-term context, long-term installation patterns and random choice.

\subsection{Norms mining}

Norms emerge where a behaviour is expected explicitly among the group or community. A few of the examples of norms are following the coding standards (e.g. avoiding the use of ternary operators in Java source code, avoiding the use of generic imports, maximum size of file, etc.), denial of permission for check-in if code does not compile, denial of permission for check-in if revised comments are not added, forking done only by project manager, etc. There are some tools already available like CheckStyle [90] and StyleCop [91], which help prohibit the violation of certain norms in projects.

Software repositories contain a huge amount of humaninteraction data, which is mostly available in e-mail discussions, bug reports, chats, commit messages and sometimes the source code as well. As in a software development team and especially open-source software community, a number of people work together; people belong to different geographical locations, backgrounds and cultures, and norms play an important role. The human-interaction data in the repositories of open-source software contains norms hidden in it, which if mined can help in smooth functioning of complex diversified open-source software development teams. Compliance to norms helps in improving the coordination and cooperation among the team [92, 93]. Dam et al [98] developed the norms life-cycle model having convention-formation, convention-modification, norms-monitoring and norms-enforcement as phases, which basically explains how the norms evolve. They also investigated how the success/failure of a project depends on the norms compliances and discussed about the requirement of norms database for open-source software development community.

In table 5, we give a summary of some of the works where we find the application of NLP for MSR. We have classified the work according to author, artefact used for mining, application, corpus used for evaluation and NLP techniques used for performing the task.

\section{Stengths and limitations of NLP in MSR}

Runeson et al [34] have stated that the classical information retrieval techniques just retrieve the results from repositories. With heterogeneous and large data, many times, the results are either empty or very large. They have claimed that the usage of NLP techniques helps in getting good results. Even though NLP is an utmost important part of mining to get the good results, NLP alone cannot perform all the tasks. Information retrieval techniques [11,99] and other techniques like ML need to be used to get good results. From the survey, it has been found that many of the authors have used either ML or information retrieval techniques along with NLP techniques $[15,41,73,74,87]$ in their works to get the desired output or to overcome the shortcomings of NLP.

Banerjee et al [43] have conducted an experiment to compare results from various techniques for duplicate bug reports identification. The results show that NLP along with execution information on Firefox yields precision of around 67-93 percent, while text analysis alone yields precision only till 50 percent. Even the models using neural networks like BM25F yielded precision till 75 percent only. This experiment shows how the NLP helps improve the results while analysing the unstructured data of software.

Nowadays, to deal with the limitations of NLP, deep learning has emerged, which uses neural networks like sequence-to-sequence attention model and feed-forward neural network model to find out the meaning of sentences in the text and perform language modelling. Deep learning is bringing revolution to the field of NLP [100]. For statistical NLP, mostly Markov models are used, but they lack capacity expressiveness [100], effective capacity and representative power. Curse of dimensionality is also one of the problems with Markov models. Deep learning helps overcome these problems.

\section{Methodology}

Our methodology involved keyword-based search and citations search. After finding out the research papers related to our purpose, we selected those papers where NLP techniques were used by reading the title and the abstract part of the research paper. While searching for the papers, we used the top 100 results as for some queries, there were more than 200 papers, and we found that the relevance of papers decreased after 50-60 results. After selecting the research papers of our interest, we classified them into the following: sentiment analysis, summarization, traceability, norms mining and mobile analytics. To ensure that the quality of paper is good, we used IEEE Xplore [102], Springer Link [103], ACM Digital Library link [104] and Google Scholar link [105]. For searching using keywords, we used the strings mentioned in table 3 .

\section{Discussion and analysis}

Figure 1 shows the trends of research in the various applications year-wise. How the research in MSR has increased from 2010 to April 2018 can be observed. 
Table 3. Keywords used for selecting the research papers.

\begin{tabular}{lc}
\hline Application & Strings used \\
\hline $\begin{array}{l}\text { Sentiment analysis } \\
\text { Summarization }\end{array}$ & $\begin{array}{c}\text { Sentiment analysis in software repositories, sentiment analysis, sentiment analysis } \\
\text { in software engineering }\end{array}$ \\
$\begin{array}{c}\text { Summarization of software artefacts, summarization in software engineering, } \\
\text { summarization of software code, summarization in software } \\
\text { Mobile analytics }\end{array}$ & $\begin{array}{c}\text { Traceability in software engineering, traceability in software repositories } \\
\text { Mobile app store mining, MSR, MSR with mobile apps, mobile app store analytics, } \\
\text { mobile software repositories with mobile apps, summarization in mobile analytics, } \\
\text { sentiment analysis in mobile apps }\end{array}$ \\
Norms mining & Norms mining, norms mining in software repositories, norms software
\end{tabular}

\begin{tabular}{|c|c|c|c|c|c|}
\hline \multirow[b]{2}{*}{ Year } & \multicolumn{5}{|c|}{ Number of papers : application wise for a particular year } \\
\hline & Summarization & $\begin{array}{l}\text { Sentiment } \\
\text { Analysis }\end{array}$ & Traceability & $\begin{array}{l}\text { Norms } \\
\text { Mining }\end{array}$ & $\begin{array}{l}\text { Mobile } \\
\text { Analytics }\end{array}$ \\
\hline till 2010 & 21 & 16 & 21 & 5 & $\mathbf{O}$ \\
\hline 2011 & 5 & 1 & 7 & 1 & $\mathrm{O}$ \\
\hline 2012 & 2 & 1 & 1 & 2 & 1 \\
\hline 2013 & 10 & 9 & 1 & 1 & 9 \\
\hline 2014 & 8 & 5 & 3 & 2 & 3 \\
\hline 2015 & 12 & 4 & 4 & 1 & 11 \\
\hline 2016 & 11 & 6 & 8 & 4 & 13 \\
\hline 2017 & 9 & 16 & 4 & $\mathrm{O}$ & 19 \\
\hline
\end{tabular}

Figure 1. Papers distribution according to the application: year-wise.

- Out of 257 papers, approximately 48 percent of the papers belong to summarization and sentiment analysis. Sentiment analysis and summarization are the most popular topics of research in the NLP field. Sentiment analysis also plays a very important role while performing summarization task.

- There is a huge increase in research works in mobile analytics from 2012 to 2015. In this survey, we have considered only the papers that involve NLP technique to perform mining-related task. The percentage increase of the works in this area is too much if we consider other techniques as well.

- Research in the field of extractive summarization [62] has reached the saturation stage after 2013 but now the research is going on in the field of abstractive summarization.

- Research in the field of sentiment analysis is increasing year by year.

- Norms mining is still a very young field of research in MSR. Only 16 papers have been published till now, presenting opportunities for research in this field.

\section{Future scope and challenges}

Here, we discuss the future research areas and the challenges that we have identified from various papers published in the recent years related to works in software repositories.

\subsection{Sentiment analysis}

It is an ongoing research topic among researchers. Lot of work has been done in this field on social sites, questionanswering sites, etc. However, from the literature review, it has been found that there is a lack of research in the field of context-based sentiment analysis.

SentiStrength, NLTK and Stanford NLP are the three dominant sentiment analysis tools available today. However, studies have shown that these tools do not give good results when applied to software engineering artefacts due to domain-related technical terms, which are frequently used in software engineering context. There are many terms in software engineering like "super", "resolve", "dead" and "block" that do not hold any sentimental polarity in domain-terms but in English dictionary, they hold sentimental polarities. Resolving these issues in this field of research is still an open problem [56]. Distinguishing the context-sensitive meanings of words and negation handling in software engineering domain is also a big challenge. There is a lack of sentiment-annotated text in software engineering for research in this field [56]. Detection of irony and sarcasm hidden in text is still an open problem in this field of research. Tourani et al [47] found that currently available sentiment analysis tools do not give good precision due to the presence of ambiguous technical terms and the difficulty of separating the neutral technical e-mails from positive and negative e-mails. They addressed the need of off-shelf tools for the purpose of sentiment analysis in software engineering context. Lin et al [125], in their 
Table 4. Paper distribution: source-wise.

\begin{tabular}{lcccccc}
\hline Link & No. of papers & Summarization papers & Sentiment analysis & Traceability & Mobile analytics & Norms mining \\
\hline Springer & 65 & 24 & 15 & 2 & 24 & 6 \\
IEEE & 87 & 31 & 25 & 22 & 9 & 7 \\
ACM & 70 & 12 & 14 & 22 & 22 & 1 \\
Others & 24 & 11 & 4 & 3 & 4 & 56 \\
Total papers found & & 78 & 58 & 49 & 16 \\
\hline
\end{tabular}

paper, addressed the concern of sentiment analysis tools in software engineering context. They analysed the results of these tools on stackOverflow sentences, comments on issue tracking sites and mobile apps, and found that the tools worked a little better with mobile app reviews and issue tracking sites but gave very less precision with stackOverflow sentences.

\subsection{Norms mining in open-source development communities}

Norms are very important for smooth functioning of a large diversified project, as they help resolve the behavioural issues among the team. From our survey, there has not been much research in this area. Many questions like how the norms differ from small projects to large projects, and how norms and the role of a person are related to each other, need to be considered; finding out under what conditions the norms emerge, how cultural differences and norms are related, how the norms adoption and compliance differ between open-source and closed-source software projects, impact of norms on stakeholders and why some norms are adopted efficiently and not some others $[93,98]$ need to be researched more. NLP plays an important role in norms mining as the human-interaction data is mostly noisy, incomplete and contains lot of domainspecific slangs, etc. NLP helps find the meaning of sentences and phrases, and the relationship between the words and sentences that help extract the norms.

\subsection{Mobile analytics}

Even though the mobile user's review data is very useful for developers, it is very massive. The reviewers talk about multiple topics and, many times, posts are incomplete [87]. In addition, the use of abbreviations, incorrect grammar, unstructured and informal nature, use of slangs and typomistakes create challenges during preprocessing of this data [82]. Handling this massive data to find out which data is important is another challenge for the developers.

\subsection{Summarization techniques}

A lot of work has been done in the field of summarization in the software engineering context. However, the questions like "what is a good summary?", "how much information should be added to a summary?", "how should a summary be evaluated?", etc. are still open questions in this field of research. There are only a few quantitative evaluation measures, like ROUGE-score and Pyramid-score, available to calculate the informativeness of the summaries. Pawar and Mago [94] used the deep semantic analysis to find the effectiveness of summaries, but it was time-consuming. There is a need for finding better ways to evaluate the summaries that not only consider the informativeness of the summaries but also various aspects like semantics, contradiction, pronoun resolution, paraphrases, etc. Not just the evaluation of summaries is a big issue but successful application of NLP techniques to create abstractive summaries is also a big challenge. There has been a lot of research in the field of extractive summarization as it is easy to perform, but research in the field of abstractive summarization is still lacking as it is very challenging because of the complexity of NLP [95]. Proper handling of pronouns considering the context, capturing of the overlapping information and sentence segmentation in conversation-related artefacts are still open problems. Representation of abstractive summarization is also one of the challenges in this field [61]. Heterogeneity [72], incompleteness of code fragments [97] and the multi-dimensional nature of software artefacts pose other challenges in the field of summarization of software artefacts.

Investigations show that abstractive summarization is more promising than extractive summarization [95]. Haiduc et al [96] showed that abstractive summarization performed better than extractive summarization. Most of the summarization techniques have been applied to textual data. However, most of the software engineering data consists of heterogeneous data consisting of code fragments from different languages, and lot of other data apart from textual data. More research is required that considers other aspects of text as well, like XML data or code snippets, which are generally embedded in the software documents [72].

Even the automatic generation of documentation for software artefacts like automatic generation of release notes, source code, commit notes, etc. is not fully explored. For example, ARENA is one approach that has been developed for release notes generation but there are many opportunities, like deciding what has to be included to the summary and what not to include, how the release notes 


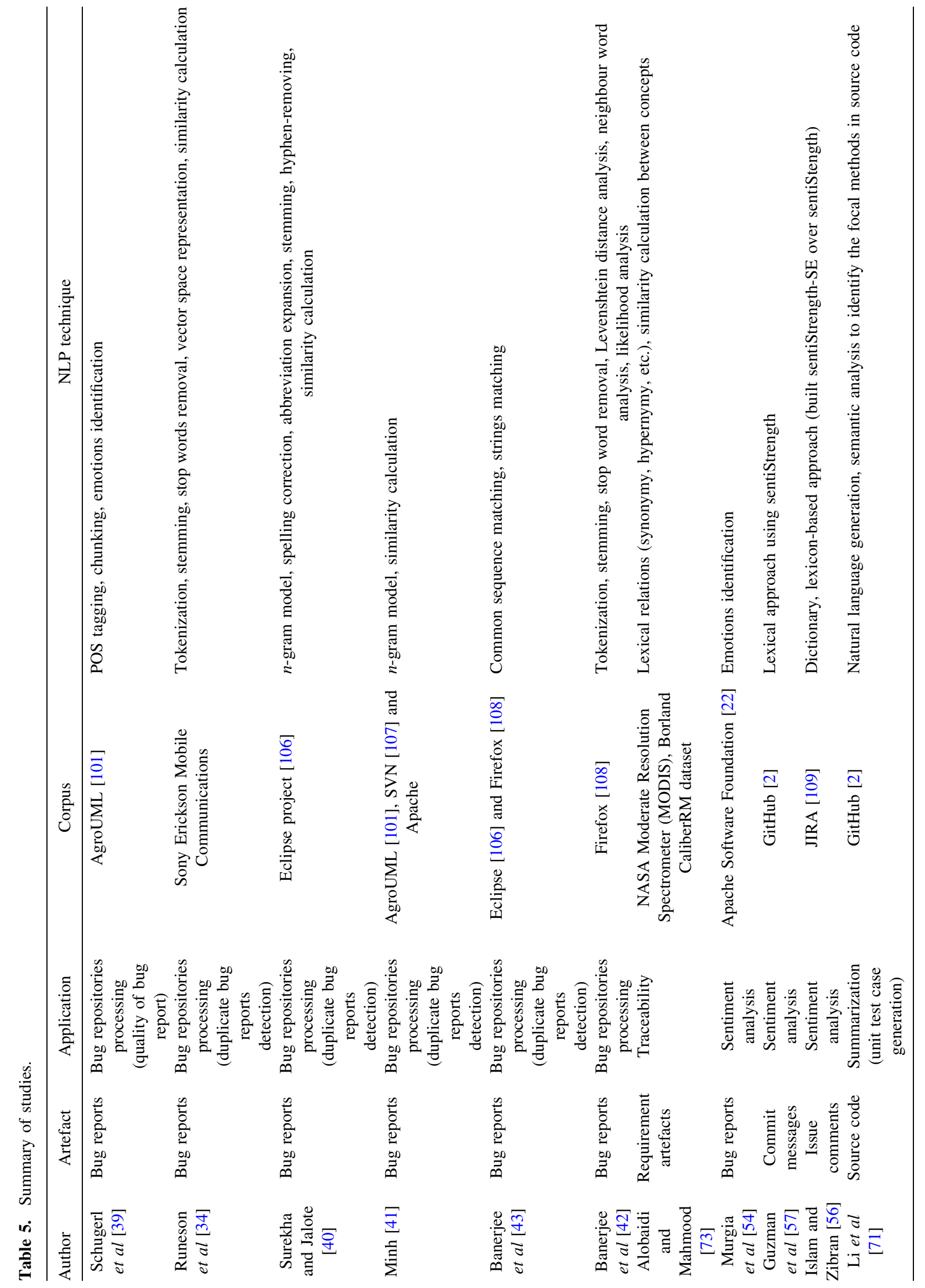




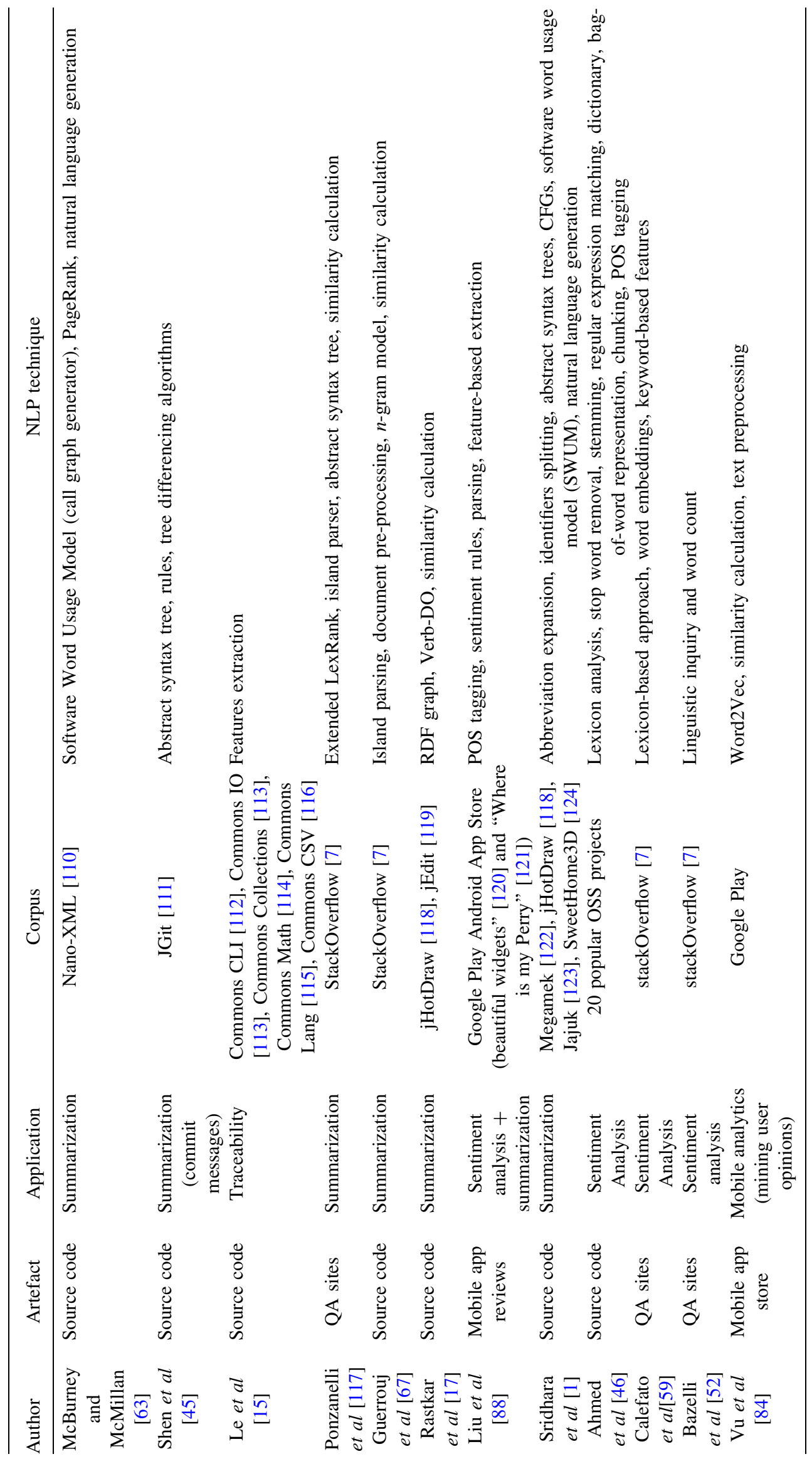


should be presented to users, [16] etc. Most of the source code summarization techniques are focused on $\mathrm{C}++$ and Java languages. More work on performing summarization for other object-oriented languages is required [62]. CrowdSourcing is one of the emerging ways of collecting information for summarization, which helps in evaluation of summaries. CrowdSourcing in the field of software summarization has been used in very small scale. More efforts on extending it are required [97]. More work on creating personalized summaries based on the role of persons is required [62].

There are not many designers of automatic documentation tools, as what should be the good characteristics for generating high-quality summaries is not yet very clear [95].

More work on creating the unit test cases summaries is required. Application of unit test case summarization on test case generation tools needs more research [71].

\section{Conclusion}

Software repositories are very complex, contain valuable information and mining them helps in supporting a number of software engineering tasks like improving the quality of software and software processes. Natural language artefacts are produced at almost each phase of software life cycle. On applying NLP techniques, these artefacts help know many important aspects related to "why" side of a software, which helps managers, developers and business stakeholders know user requirements, loop holes in their projects, team collaboration style, user's feedback and get continuous inputs for the betterment of their project. Heterogeneous data present in these repositories requires knowledge about the semantics and context. NLP simplifies the processing. In this paper, we focused on the application of NLP and its future scope in the field of MSR. We have also discussed the tools, techniques and the challenges lying in this field of research.

\section{References}

[1] Sridhara G, Hill E, Muppaneni D, Pollock L and Vijay Shanker K 2010 Towards automatically generating summary comments for Java methods. In: Proceedings of the IEEE/ACM International Conference on Automated Software Engineering, pp. 43-52, https://doi.org/10.1145/ 1858996.1859006

[2] https://github.com/

[3] https://sourceforge.net/

[4] https://code.google.com/

[5] https://www.bugzilla.org/about/

[6] https://academia.stackexchange.com/

[7] https://stackoverflow.com/

[8] https://octoverse.github.com/
[9] Chen T H, Thomas S W and Hassan A E 2015 A survey on the use of topic models when mining software repositories. Empirical Software Engineering 21: 1843-1919

[10] White M, Vendome C, Vasquez M L and Poshyvanyk D 2015 Toward deep learning software repositories. In: Proceedings of the 12th Working Conference on Mining Software Repositories, pp. 334-345, https://doi.org/10. 1109/MSR.2015.38

[11] Haiduc S, Arnaoudov V, Marcus A and Antoniol G 2016 The use of text retrieval and natural language processing in software engineering. In: Proceedings of the 38th IEEE International Conference on Software Engineering, pp. 898-899, https://doi.org/10.1145/2889160.2891053

[12] Nazir F, Butt W H, Anwar M W and Khan Khattak M A 2017 The applications of natural language processing (NLP) for software engineering-a systematic literature review. In: Lecture Notes in Electrical Engineering, 424: 485-493

[13] Hassan A E 2008 The road ahead for mining software repositories. In: Proceedings of FoSM 2008, pp. 48-57, https://doi.org/10.1109/FOSM.2008.4659248

[14] Rastkar S, Murphy G C and Murray G 2014 Automatic summarization of bug reports. IEEE Transactions on Software Engineering 40: 366-380

[15] Le T D B, Vasquez M L, Lo D and Poshyvanyk D 2015 RCLinker: automated linking of issue reports and commits leveraging rich contextual information. In: Proceedings of the 23rd International Conference on Program Comprehension, pp. 36-47, https://doi.org/10.1109/ICPC.2015.13

[16] Moreno L, Bavota G and Penta M D 2016 ARENA: an approach for the automated generation of release notes. IEEE Transactions on Software Engineering 43: 106-127

[17] Rastkar S, Murphy G C, Moreno L and Bradley A W J 2011 Generating natural language summaries for crosscutting source code concerns. In: Proceedings of the 27th IEEE International Conference on Software Maintenance (ICSM), pp. 103-112, https://doi.org/10.1109/ICSM.2011. 6080777

[18] https://www.statista.com/statistics/276623/number-of-appsavailable-in-leading-app-stores/

[19] http://www.businessofapps.com/data/app-statistics/

[20] Kao A and Poteet S R (Eds.) 2007 Natural language processing and text mining. London: Springer, https://doi. org/10.1007/978-1-84628-754-1

[21] LEBRET R P 2016 Word embeddings for natural language processing. Ph.D. Thesis, Ecole polytechnique federale de Lausanne, Chapter 3

[22] https://projects.apache.org/

[23] https://opennlp.apache.org/

[24] https://stanfordnlp.github.io/CoreNLP/

[25] https://www.nltk.org/

[26] https://gate.ac.uk/

[27] https://spacy.io/

[28] https://github.com/collab-uniba/Emotion/

[29] http://mallet.cs.umass.edu/

[30] https://github.com/tensorflow/tensorflow

[31] https://github.com/Microsoft/cntk

[32] https://github.com/Theano/Theano

[33] https://keras.io/

[34] Runeson P, Alexandersson M and Nyholm O 2007 Detection of duplicate defect reports using natural language 
processing. In: Proceedings of the 29th International Conference on Software Engineering, https://doi.org/10. 1109/ICSE.2007.32

[35] Moawad I F and Aref M 2012 Semantic graph reduction approach for abstractive text summarization. In: Proceedings of the Seventh International Conference on Computer Engineering and Systems, pp. 132-138, https://doi.org/10. 1109/ICCES.2012.6408498

[36] Dohare S, Karnick H and Gupta V 2017 Text summarization using abstract meaning representation. Computation and LanguagearXiv:1706.01678v3

[37] https://en.wikipedia.org/wiki/Word-sense_disambiguation

[38] Saberi B and Saad S 2017 Sentiment analysis or opinion mining: a review. International Journal of Advanced Science Engineering Information Technology 7: 1660-1667

[39] Schugerl P, Rilling J and Charland P 2008 Mining bug repositories: a quality assessment. In: Proceedings of the 38th IEEE International Conference on Software Engineering, pp. 1105-1110, https://doi.org/10.1109/CIMCA. 2008.63

[40] Sureka A and Jalote P 2010 Detecting duplicate bug report using character n-gram-based features. In: Proceedings of the Asia Pacific Software Engineering Conference, pp. 366-374, https://doi.org/10.1109/APSEC.2010.49

[41] Minh P N 2014 An approach to detecting duplicate bug reports using n-gram features and cluster shrinkage technique. International Journal of Scientific and Research Publications 4: 1-8

[42] Banerjee S, Musgrove J and Cukic B 2012 Handling language variations in open source bug reporting systems. In: Proceedings of the 23rd IEEE International Symposium on Software Reliability Engineering Workshops, pp. 325-330, https://doi.org/10.1109/ISSREW.2012.85

[43] Banerjee S, Cukic B and Adjeroh D 2012 Automated duplicate bug report classification using subsequence matching. In: Proceedings of the 14th IEEE International Symposium on High-Assurance Systems Engineering, pp. 74-81, https://doi.org/10.1109/HASE.2012.38

[44] Bavota G 2016 Mining unstructured data in software repositories: current and future trends. In: Proceedings of the 23rd IEEE International Conference on Software Analysis, Evolution, and Re-engineering (SANER), pp. 1-12, https://doi.org/10.1109/SANER.2016.47

[45] Shen J, Sun X, Li B, Yang H and Hu J 2016 On automatic summarization of what and why information in source code changes. In: Proceedings of the 40th Annual Computer Software and Applications Conference, pp. 103-112, https://doi.org/10.1109/COMPSAC.2016.162

[46] Ahmed T, Bosu A and Iqbal A 2017 SentiCR: a customized sentiment analysis tool for code review interactions. In: Proceedings of the 32nd IEEE/ACM International Conference on Automated Software Engineering (ASE), pp. 106-111, https://doi.org/10.1109/ASE.2017.8115623

[47] Tourani P, Jiang Y and Adams B 2014 Monitoring sentiment in open source mailing lists: exploratory study on the apache ecosystem. In: Proceedings of the 24th Annual International Conference on Computer Science and Software Engineering, CASCON '14, pp. 34-44

[48] Goul M, Marjanovic O, Baxley S and Vizecky K 2012 Managing the enterprise business intelligence app store: sentiment analysis supported requirements engineering. In: Proceedings of the 45th Hawaii International Conference on System Sciences, pp. 4168-4177, https://doi.org/10. 1109/HICSS.2012.421

[49] Carreno L V G and Winbladh K 2013 Analysis of user comments: an approach for software requirements evolution. In: Proceedings of ICSE 2013, pp. 582-591, https:// doi.org/10.1109/ICSE.2013.6606604

[50] https://tomcat.apache.org/tomcat-7.0-doc/appdev/deploy ment.html

[51] https://ant.apache.org/manual/api/org/apache/tools/ant/task defs/optional/unix/Symlink.html

[52] Bazelli B, Hindle A and Stroulia E 2013 On the personality traits of StackOverflow users. In: Proceedings of the 29th IEEE International Conference on Software Maintenance, pp. 460-463, https://doi.org/10.1109/ICSM.2013.72

[53] Ortu M, Adams B, Destefanis G, Tourani P, Marchesi M and Tonelli R 2015 Are bullies more productive? Empirical study of affectiveness vs. issue fixing time. In: Proceedings of the 12th Working Conference on Mining Software Repositories, 480-483, https://doi.org/10.1109/MSR.2015.35

[54] Murgia A, Tourani P, Adams B and Ortu M 2014 Do developers feel emotions? An exploratory analysis of emotions in software artifacts. In: Proceedings of the International Conference on Mining Software Repositories (MSR), pp. 262-271, https://doi.org/10.1145/2597073. 2597086

[55] Ortu M, Murgia A and Destefanis G 2016 The emotional side of software developers in JIRA. In: Proceedings of the 13th Working Conference on Mining Software Repositories, pp. 480-483, https://doi.org/10.1145/2901739.2903505

[56] Islam M R and Zibran M F 2017 Leveraging automated sentiment analysis in software engineering. In: Proceedings of the 14th International Conference on Mining Software Repositories (MSR), pp. 203-214, https://doi.org/10.1109/ MSR.2017.9

[57] Guzman E, Azócar D and Li Y 2014 Sentiment analysis of commit comments in GitHub: an empirical study. In: Proceedings of the Working Conference on Mining Software Repositories, pp. 352-355, https://doi.org/10.1145/ 2597073.2597118

[58] Sinha V, Lazar A and Sharif B 2016 Analyzing developer sentiment in commit logs. In: Proceedings of the 13th IEEE/ACM Working Conference on Mining Software Repositories, pp. 520-523, https://doi.org/10.1145/ 2901739.2903501

[59] Calefato F, Lanubile F, Maiorano F and Novielli N 2017 Sentiment polarity detection for software development. Empirical Software Engineering 23: 1352-1382

[60] Buse R P L and Weimer W R Automatic documentation inference for exceptions. In: Proceedings of the 2008 International Symposium on Software Testing and Analysis, pp. 273-282, https://doi.org/10.1145/1390630.1390664

[61] Moratanch N and Chitrakala S 2016 A survey on abstractive text summarization. In: Proceedings of the International Conference of Circuit, Power and Computing Technologies (ICCPCT), https://doi.org/10.1109/ICCPCT. 2016.7530193

[62] Gupta S and Gupta S K 2017 Summarization of software artifacts: a review. International Journal of Computer Science and Information Technology 5: 165-187 
[63] McBurney P W and McMillan C 2015 Automatic source code summarization of context for Java methods. IEEE Transactions on Software Engineering 42: 103-119

[64] Nithya R and Arunkumar A 2016 Summarization of bug reports using feature extraction. International Journal of Computer Science and Mobile Computing 5: 268-273

[65] Lotufo R, Malik Z and Czarnecki K 2013 Modelling the Hurried bug report reading process to summarize bug reports. In: Proceedings of the 28th IEEE International Conference on Software Maintenance (ICSM), pp. 430-439, https://doi.org/10.1109/ICSM.2012.6405303

[66] Haiduc S, Aponte J, Moreno L and Marcus A 2010 On the use of automated text summarization techniques for summarizing source code. In: Proceedings of the 17th Working Conference on Reverse Engineering, pp. 35-44, https://doi. org/10.1109/WCRE.2010.13

[67] Guerrouj L, Bourque D and Rigby P C 2015 Leveraging informal documentation to summarize classes and methods in context. In: Proceedings of the 37th IEEE International Conference on Software Engineering, pp. 639-642, https:// doi.org/10.1109/ICSE.2015.212

[68] Chitti Babu K, Kavitha C and SankarRam N 2016 Entity based source code summarization (EBSCS). In: Proceedings of the 3rd International Conference on Advanced Computing and Communication Systems, https://doi.org/10. 1109/ICACCS.2016.7586385

[69] Cortes-Coy L F, Linares-Vasquez M and Aponte J 2014 On automatically generating commit messages via summarization of source code changes. In: Proceedings of the 14th International Working Conference on Source Code Analysis and Manipulation (SCAM), pp. 275-284, https://doi. org/10.1109/SCAM.2014.14

[70] Linares-Vasquez M, Cortes-Coy L F and Aponte J 2015 ChangeScribe: a tool for automatically generating commit messages. In: Proceedings of the 37th IEEE International Conference on Software Engineering (ICSE), pp. 257-277, https://doi.org/10.1109/ICSE.2015.229

[71] Li B, Vendome C, Vasquez M L, Poshyvanyk D and Kraft N A 2016 Automatically documenting unit test cases. In: Proceedings of the IEEE International Conference on Software Testing, Verification and Validation, pp. 341-352, https://doi.org/10.1109/ICST.2016.30

[72] Ponzanelli L, Mocci A and Lanza M 2015 Summarizing complex development artifacts by mining heterogeneous data. In: Proceedings of the 12th IEEE/ACM Working Conference on Mining Software Repositories (MSR), pp. 401-405, https://doi.org/10.1109/MSR.2015.49

[73] Alobaidi M and Mahmood K 2015 Semantic approach for traceability link recovery using uniform resource identifier. In: Proceedings of the International Conference on Software Engineering Research and Practice, pp. 190-195

[74] Aponte J and Marcus A 2011 Improving traceability link recovery methods through software artifact summarization. In: Proceedings of TEFSE 2011, pp. 46-49, ACM 978-14503-0589-1/11/05

[75] Arunthavanathan A, Shanmugathasan S, Ratnavel S, Thiyagarajah V, Perera I et al Support for traceability management of software artefacts using Natural Language Processing. In: Proceedings of the Moratuwa Engineering Research Conference (MERCon), pp. 18-23, https://doi. org/10.1109/MERCon.2016.7480109
[76] https://wordnet.princeton.edu/

[77] https://propbank.github.io/

[78] https://verbs.colorado.edu/verbnet/

[79] Liu F, Flanigan J, Thomson S, Sadeh N and Smith N A 2015 Toward abstractive summarization using semantic representations. In: Proceedings of Human Language Technologies: The 2015 Annual Conference of the North American Chapter of the ACL, pp. 1077-1086

[80] Gupta S and Gupta S K 2019 Abstractive summarization: an overview of the state of the art. Expert Systems with Applications 121: 49-65

[81] Santos F L D and Ladeira M The role of text pre-processing in opinion mining on a social media language dataset. In: Proceedings of the Brazilian Conference on Intelligent Systems, pp. 50-54, https://doi.org/10.1109/BRACIS.2014. 20

[82] Mcilroy S, Ali N, Khalid H and Hassan A E 2015 Analyzing and automatically labelling the types of user issues that are raised in mobile app reviews. Empirical Software Engineering 21: 1067-1106

[83] Hu H, Wang S, Bezemer C P and Hassan A E 2018 Studying the consistency of star ratings and reviews of popular free hybrid Android and iOS apps. Empirical Software Engineering 24: 7-32

[84] Vu P M, Nguyen T T, Pham H V and Nguyen T T 2015 Mining user opinions in mobile app reviews: a keywordbased approach. In: Proceedings of the 30th IEEE/ACM International Conference on Automated Software Engineering (ASE), https://doi.org/10.1109/ASE.2015.85

[85] Zhang L, Huang X Y, Jiang J and Hu Y K 2017 CSLabel: an approach for labelling mobile app reviews. Journal of Computer Science and Technology 32: 1076-1089

[86] Iacob C and Harrison R 2013 Retrieving and analyzing mobile apps feature requests from online reviews. In: Proceedings of the 10th Working Conference on Mining Software Repositories (MSR), pp. 41-44, https://doi.org/10. 1109/MSR.2013.6624001

[87] Gao C, Xu H, Hu J and Zhou Y 2015 AR-tracker: track the dynamics of mobile apps via user review mining. Proceedings of the IEEE Symposium on Service-Oriented System Engineering, pp. 4168-4177, https://doi.org/10.1109/ SOSE.2015.13

[88] Liu J, Sarkar M K and Chakraborty G 2013 Feature-based sentiment analysis on android app reviews using SAS text. In: Proceedings of SAS Global Forum 2013, https://doi.org/ 10.1.1.381.3525

[89] Cheng V C, Chen L, Cheung W K and Fok C K 2017 A heterogeneous hidden Markov model for mobile app recommendation. Knowledge Information Systems 57: 207-228

[90] http://checkstyle.org/eclipse-cs/

[91] https://archive.codeplex.com/?p=stylecop

[92] Cheng V C, Chen L, Cheung W K and Fok C K 2011 Norm creation, spreading and emergence: a survey of simulation models of norms in multi-agent systems. Multiagent and Grid Systems-An International Journal 7: 21-54

[93] Savarimuthu B T and Dam H K 2013 Towards mining norms in open source software repositories. In: ADMI Revised Selected Papers of the 9th International Workshop on Agents and Data Mining Interaction, pp. 26-39, https:// doi.org/10.1007/978-3-642-55192-53 
[94] Pawar A and Mago V 2018 Calculating the similarity between words and sentences using a lexical database and corpus statistics. IEEE Transactions on Knowledge and Data Engineering 18: 1-4

[95] Khan A and Salim N 2014 A review on abstractive summarization methods. Journal of Theoretical and Applied Information Technology 59: 64-72

[96] Haiduc S, Aponte J, Moreno L and Marcus A On the use of automated text summarization techniques for summarizing source code. In: Proceedings of the 17th Working Conference on Reverse Engineering (WCRE), pp. 35-44, https:// doi.org/10.1109/WCRE.2010.13

[97] Jiang N N H, Gao G, Zhang T, Li X and Ren Z 2016 Source code fragment summarization with small-scale crowdsourcing based features. Frontiers of Computer Science 10: 504-517

[98] Dam H K, Savarimuthu B T R and Avery D 2015 Mining software repositories for social norms. In: Proceedings of the 37th IEEE/ACM International Conference on Software Engineering, pp. 284-290, https://doi.org/10.1109/ICSE.2015.209

[99] Nazir F, Butt W H, Anwar M W and Khan Khattak M A 2017 The application of natural language processing (NLP) for software requirement engineering - a systematic review. Lecture Notes in Electrical Engineering 424: 485-493

[100] White M, Vendome C, Linares-Vasquez M and Poshyvanyk D 2015 Toward deep learning software repositories. In: Proceedings of the 12th Working Conference on Mining Software Repositories, pp. 334-345, https://doi.org/10. 1109/MSR.2015.38

[101] https://argouml.en.softonic.com/

[102] https://ieeexplore.ieee.org/Xplore/home.jsp

[103] https://link.springer.com/
[104] https://dl.acm.org/

[105] https://scholar.google.co.in/

[106] https://www.eclipse.org/eclipse/

[107] https://subversion.apache.org/

[108] https://www-archive.mozilla.org/projects/firefox/

[109] https://developer.atlassian.com/docs/

[110] http://nanoxml.sourceforge.net/orig/

[111] https://www.eclipse.org/jgit/

[112] http://commons.apache.org/proper/commons-cli/

[113] http://commons.apache.org/proper/commons-io/

[114] https://commons.apache.org/proper/commons-math/

[115] https://commons.apache.org/proper/commons-lang/

[116] https://commons.apache.org/proper/commons-csv/

[117] Ponzanelli L, Mocci A and Lanza M 2015 Summarizing complex development artifacts by mining heterogeneous data. In: Proceedings of the 12th Working Conference on Mining Software Repositories, pp. 401-405, https://doi.org/ 10.1109/MSR.2015.49

[118] http://www.jhotdraw.org/

[119] http://www.jedit.org/

[120] https://play.google.com/store/apps/details?id=com.levelup. beautifulwidgets\&hl=en_IN

[121] https://where-is-my-perry.en.uptodown.com/android

[122] https://www.megamek.org/

[123] https://www.openhub.net/p/p_5944

[124] http://www.sweethome3d.com/

[125] Lin B, Zampetti F, Bavota G, Penta M D, Lanza M and Oliveto R 2018 Sentiment analysis for software engineering: how far can we go? In: Proceedings of the 40th International Conference on Software Engineering, pp. 94-104, https://doi.org/10.1145/3180155.3180195 